Abstracta Iranica Abstracta Iranica

Revue bibliographique pour le domaine irano-aryen

Volume 40-41 | 2019

Comptes rendus des publications de 2017-2018

\title{
Ghazaleh Vafaeian. Progressives in use and contact: A descriptive, areal and typological study with special focus on selected Iranian languages
}

\section{Murad Suleymanov}

\author{
(2) OpenEdition \\ Journals \\ Édition électronique \\ URL : http://journals.openedition.org/abstractairanica/51052 \\ DOI : 10.4000/abstractairanica.51052 \\ ISBN : 1961-960X \\ ISSN : 1961-960X \\ Éditeur : \\ CNRS (UMR 7528 Mondes iraniens et indiens), Éditions de l'IFRI
}

\section{Référence électronique}

Murad Suleymanov, « Ghazaleh Vafaeian. Progressives in use and contact: A descriptive, areal and typological study with special focus on selected Iranian languages », Abstracta Iranica [En ligne], Volume 40-41 | 2019, document 4, mis en ligne le 30 décembre 2019, consulté le 24 avril 2021. URL : http:// journals.openedition.org/abstractairanica/51052 ; DOI : https://doi.org/10.4000/abstractairanica. 51052

Ce document a été généré automatiquement le 24 avril 2021.

Tous droits réservés 


\title{
Ghazaleh Vafaeian. Progressives in use and contact: A descriptive, areal and typological study with special focus on selected Iranian languages
}

\author{
Murad Suleymanov
}

\section{RÉFÉRENCE}

Ghazaleh Vafaeian. Progressives in use and contact: A descriptive, areal and typological study with special focus on selected Iranian languages. Stockholm: Stockholm University, 2018, xiv+275 p. <http://su.diva-portal.org/smash/get/diva2:1200741/FULLTEXT01.pdf>

1 Cette thèse, soutenue en 2018, est une étude approfondie de l'emploi du progressif, une catégorie grammaticale définie par l'A. comme signalant que "a dynamic event is ongoing at a specific time» (p. 1), dans certaines langues iraniennes ainsi que d'un point de vue typologique. La thèse contient une introduction générale assez dense, qui présente la terminologie associée au progressif ainsi qu'un aperçu typologique de cette catégorie telle qu'elle est décrite dans les ouvrages publiés à ce jour. Les chapitres suivants présentent les résultats des recherches menées par l'A., qui sont ensuite reliées par les analyses de leurs traits communs dans les chapitres intermédiaires. Ces résultats portent sur l'emploi du progressif en persan et sur les catégories progressives dans les langues d'Iran du nord et d'Azerbaïdjan du sud.

Le corpus parallèle étudié par l'A. pour la première partie comprend les traductions de la Bible et les transcriptions des conférences TED ( Technology, Entertainment and Design »). Bien que les premières contiennent un nombre beaucoup plus grand de langues que les dernières et soient caractérisées par un genre très différent, l'A. arrive aux mêmes conclusions concernant l'usage du progressif, à savoir notamment que dans les deux corpus, d'une part, l'emploi au présent est plus courant que l'emploi au passé, 
et d'autre part, que le progressif est le plus souvent utilisé afin de focaliser une action en cours.

3 La partie suivante propose un exposé détaillé (sur la base d'un questionnaire rempli par des locuteurs natifs mais aussi d'un corpus spontané) sur les fonctions de la construction progressive du persan, qui repose sur le verbe dāštan 'avoir' et qui incluent la focalisation d'une action en cours (y compris pour des raisons émotives), l'implication ou l'engagement dans une action et des fonctions « retombées » dans les domaines du non-présent et du non-passé, comme le proximatif (pour les achèvements et les événements possibles à éviter) et le futur (pour les verbes de mouvement).

4 Par la suite, l'A. combine les données relevées du corpus parallèle (en s'appuyant aussi sur l'aide de locuteurs natifs) avec les données du persan étudiées dans le chapitre précédent, et essaie de déterminer d'éventuels points communs dans l'emploi de cette catégorie dans les langues sélectionnées. Elle constate que les langues partagent la sémantique continue, proximative et itérative du progressif alors que la sémantique future est visiblement plus restreinte. L'A. conclut que le chemin de grammaticalisation des constructions progressives, qui n'est pas identique à travers les langues étudiées, explique la dissonance dans leur sémantique et la difficulté d'identification des emplois principaux.

5 La partie la plus vaste de la thèse est consacrée aux catégories progressives dans les variétés dites "iraniennes du nord-ouest", traditionnellement regroupées comme dialectes du mazandarani, du guilaki, du talyshi et du tati, et parlées le long de la côte caspienne. Après un résumé étendu des formes et des fonctions des progressifs à partir des sources scientifiques, l'A. démontre une capacité très forte des constructions progressives à être calquées d'une variété à une autre dans une situation de contact intense. Elle précise que les progressifs font partie des domaines dans lesquels une (non-)attestation dans une variété de prestige (qui fait souvent l'objet de descriptions linguistiques) ne permet pas d'apprécier l'ensemble des attestations dans les variétés périphériques ; les données dialectales détaillées sont donc d'une importance capitale pour la reconstruction du chemin de diffusion des calques. L'A. ajoute à cette conclusion le fait que l'abondance des formes progressives qui possèdent les mêmes fonctions est conforme au phénomène typologiquement connu de l'existence de constructions concurrentes au début d'un processus de grammaticalisation et leur disparition ultérieure une fois le processus achevé.

6 Dans le chapitre final, l'A. se pose la question de l'origine de la construction progressive en persan, tenant compte des conclusions sur les phénomènes de contact dans la zone caspienne. Elle présente deux scénarios admissibles, avec les avantages et les inconvénients de chaque scénario, puis elle admet que sans corpus historique provenant des langues avoisinantes au persan ainsi que sans la diachronie du registre parlé (qui contient beaucoup plus d'exemples de formes progressives que le registre soutenu), il est impossible d'arriver à une conclusion définitive concernant l'origine de la construction avec dāštan en persan.

7 Cette thèse est une contribution précieuse à l'étude typologique du progressif grâce au fait qu'elle met en œuvre une variété de méthodes : une comparaison interlinguistique via un corpus écrit parallèle, des enquêtes auprès des locuteurs natifs et des données qui relèvent de plusieurs travaux publiés. Cela permet à l'A. de mieux saisir et décrire les champs et les sous-champs sémantiques associés à l'emploi de cette catégorie afin de compléter son profil typologique. La thèse est aussi remarquable du fait qu'une 
partie des données persanes citées par l'A. provient non pas d'exemples imaginés ou forgés, comme l'on en trouve souvent dans les ouvrages existants sur ce sujet, mais du discours naturel et spontané, ce qui permet de catégoriser les fonctions du progressif dans cette langue avec plus de précision. Pour finir, la thèse présente une excellente synthèse des données sur les variétés iraniennes peu connues de l'aire caspienne concernant les formes et l'emploi du progressif, et elle met en valeur le rôle du contact linguistique dans le développement et la diffusion des constructions spécifiques.

Progressives in use and contact présente une recherche qui ouvre l'accès à une investigation plus approfondie des particularités des progressifs dans l'aire linguistique Iran-Anatolie-Caucase du point de vue à la fois synchronique et diachronique et en incluant aussi les variétés non-iraniennes de ladite aire. Le cadre typologique proposé par l'A. pourrait servir de base pour une analyse détaillée, avec la possibilité d'élargir ce cadre ou de le préciser davantage en fonction de la description de propriétés jusqu'ici inconnues.

\section{AUTEURS}

\section{MURAD SULEYMANOV}

Doctorant à l'EPHE-PSL, Paris 